\title{
miR-138 suppresses the proliferation, metastasis and autophagy of non-small cell lung cancer by targeting Sirt1
}

\author{
ZAITING YE $^{1}$, BINGMU FANG ${ }^{1}$, JIONGWEI PAN ${ }^{1}$, NING ZHANG ${ }^{1}$, \\ JINWEI HUANG ${ }^{2}$, CONGYING XIE $^{3}$, TIANZHENG LOU ${ }^{1}$ and ZHUO CAO ${ }^{1}$ \\ ${ }^{1}$ The Sixth Affiliated Hospital of Wenzhou Medical University/Lishui People's Hospitlal, Lishui, Zhejiang 323000; \\ ${ }^{2}$ The Central Hospital of Lishui City, Lishui, Zhejiang 323000; ${ }^{3}$ The First Affiliated Hospital \\ of Wenzhou Medical University, Lishui, Zhejiang 323000, P.R. China
}

Received October 14, 2016; Accepted April 11, 2017

DOI: $10.3892 / o r .2017 .5619$

\begin{abstract}
The present study determined the role and mechanism of miR-138 in non-small cell lung cancer (NSCLC). In total, 45 freshly resected clinical NSCLC tissues were collected. The expression of miR-138 in tissues and cell lines were determined by real-time quantitative PCR. miR-138 mimics were transfected into A549 and Calu-3 cells in vitro, and then the effects of miR-138 on lung cancer cell proliferation, cell cycle, invasion and metastasis were investigated by CCK-8 assay, Transwell and flow cytometry, respectively. The protein expression of the potential target gene Sirtl in lung cancer cells were determined by western blot analysis. DualLuciferase reporter assay was performed to further confirm whether Sirtl was the target gene of miR-138. The expression of miR-138 was significantly lower in lung cancer tissues and was negatively correlated to the differentiation degree and lymph node metastasis of lung cancer. In vitro experiment results showed that miR-138 inhibited lung cancer cell proliferation, invasion and migration. It was verified that miR-138 could downregulate Sirt1 protein expression, inhibit epithelialmesenchymal transition (EMT), decrease the activity of AMPK signaling pathway and elevate mTOR phosphorylation level. Dual-Luciferase reporter assay demonstrated that miR-138 could directly regulate Sirt1. Downregulation of Sirtl alone can also cause the same molecular and biological function changes. Western blot analysis and confocal micros-
\end{abstract}

Correspondence to: Dr Zhuo Cao, Department of Respiratory Medicine, The Sixth Affiliated Hospital of Wenzhou Medical University/Lishui People's Hospital, Lishui, Zhejiang 323000, P.R. China

E-mail: caozhuo001@126.com

Dr Tianzheng Lou, Department of ICU, The Sixth Affiliated Hospital of Wenzhou Medical University/Lishui People's Hospital, Lishui, Zhejiang 323000, P.R. China

E-mail:1s2780162@163.com

Key words: miR-138, non-small cell lung cancer, autophagy, mTOR, signaling pathway copy results indicated that overexpression of miR-138 or interference of Sirt1 expression could inhibit lung cancer cell autophagy activity possibly through AMPK-mTOR signaling pathway. miR-138 plays a tumor suppressor function in lung cancer. It may inhibit the proliferation, invasion and migration of lung cancer through downregulation of Sirt1 expression and activation of cell autophagy. The downregulation of miR-138 is closely related to the development of lung cancer.

\section{Introduction}

Lung cancer is currently one of the malignancies with the fastest growing morbidity and mortality worldwide. The statistical results of American Cancer Society (ACS) showed that lung cancer accounted for $28 \%$ of tumor-related death and caused a major threat to human health and life (1-3). Lung cancer is mainly classified into two tissue subtypes as nonsmall cell lung cancer (NSCLC) and small cell lung cancer (SCLC). NSCLC accounts for $~ 80 \%$ of lung cancer, including adenocarcinoma, squamous cell carcinoma and large cell carcinoma (4). Currently, surgical resection combined with chemotherapy and radiotherapy is still the main means of clinical treatment for lung cancer. However, the patients have poor prognosis and 5-year survival rate is $\sim 15 \%$ (5). Metastasis is one of the major causes of death in patients with lung cancer. The molecular mechanism of invasion and metastasis of NSCLC is still unclear; therefore, identification of the key genes of metastasis is of great significance to the clinical diagnosis and treatment of NSCLC (6).

Autophagy widely exists in all eukaryotic cells $(7,8)$. Existing studies have shown that autophagy has a 'doubleedged sword' effect on tumor cells. On one hand, autophagy can suppress early tumorigenesis; on the other hand, autophagy can promote tumor cell resistance to chemotherapy, invasion and metastasis $(9,10)$. The regulation mechanism of autophagy in NSCLC is still unclear. miRNAs are a group of highly conserved endogenous non-coding small RNA molecules with a length of 18-23 bases. miRNAs bind to the 3'-UTR region of the target gene to form a silencing complex, and thus inhibit the protein translation of the target gene $(11,12)$. miRNA molecules are extensively involved in tumor cell proliferation, apoptosis, invasion and metastasis and also have important 
regulation effects on the tumor cell autophagy $(13,14)$. For example, miR-487b-5p can inhibit the resistance of NSCLC to temozolomide through downregulating the expression of the autophagy related gene LAMP2 (15). Tomasetti et al (16) showed that miR-126 promoted autophagy of malignant mesothelioma cells by regulating cell metabolism. Nyhan et al (17) indicated that miR-193b was able to promote esophageal cancer cell autophagy and autophagy-like death. Therefore, miRNAs may play important roles in regulating cancer cell autophagy.

miR-138 is a recently discovered miRNA molecule, including two subtypes of miR-138-1 and miR-138-2 that respectively locates at $3 \mathrm{p} 21$ and $16 \mathrm{q} 13$ (18). The mature miRNA molecules of these two subtypes have a consensus sequence. It has been reported that miR-138 plays a role as tumor suppressor gene in many tumors. For instance, miR-138 plays a role as tumor suppressor gene in osteosarcoma by regulating DEC2 expression (19). It also can inhibit tumor cell proliferation by downregulation of C-Met (20). To date, the role of miR-138 in NSCLC is rarely reported. In the present study, the expression, effect and mechanism of miR-138 in NSCLC were investigated.

\section{Materials and methods}

Tissue sample collection. A total of 45 freshly resected NSCLC and corresponding adjacent tissues were collected from January 2014 to December 2015 in the Sixth Affiliated Hospital of Wenzhou Medical University. All samples were diagnosed as NSCLC by the Pathology Department. Patient ages were 29-68, average of $44.7 \pm 1.5$ years. None of the patients had been administered adjuvant therapy before the operation. According to the clinical and pathological features, there were 28 patients with lymph node metastasis (N1) and 17 patients without lymph node metastasis (N0). TNM staging is based on the TNM staging criteria for non-small cell lung cancer (2003 Edition) established by the American Joint Committee on Cancer (AJCC) (21). According to this criterion, there were 10 cases of stage I, 16 cases of stage II, 10 cases of stage III and 9 cases of stage IV. According to the differentiation degree, there were 19 well-differentiated, 17 moderately differentiated and 9 cases of low differentiation. All tissues were frozen by liquid nitrogen and stored at $-80^{\circ} \mathrm{C}$ after resected. Prior written and informed consent were obtained from every patient and the study was approved by the ethics review board of Wenzhou Medical University.

Reagents. miR-138 mimics and negative control were purchased from Guangzhou RiboBio, Co., Ltd. (Guangzhou, China). ShR-silent information transcriptional regulator (Sirt1) lentivirus and negative control were constructed by Hanbio Co., Ltd. (Shanghai, China). Rabbit anti-human Sirt1 polyclonal antibodies were purchased from Abcam plc. (Boston, MA, USA). Antibodies of AMPK, phosphorylated AMPK, E-cadherin, vimentin, mTOR, phosphorylated mTOR, LC3B and p62 were purchased from Cell Signaling Technology, Inc. (Danvers, MA, USA). TRIzol and Lipofectamine 2000 were purchased from Invitrogen (Carlsbad, CA, USA). TIANScript RT kit for reverse transcription and Quant One-Step qRT-PCR kit were purchased from Tiangen Biotech, Co., Ltd. (Beijing,
China). Transwell assay kit was purchased from Corning Co. (Boston, MA, USA).

Cell culture. Human lung cancer cell lines A549, Calu-3 and PAa were all purchased from the American Type Culture Collection (ATCC; Manassas, VA, USA). NCl-H292, $\mathrm{NCl}-\mathrm{H} 1299, \mathrm{NCl}-\mathrm{H} 1650$ and NCl-H1975 were all purchased from Shanghai Institutes for Biological Sciences (Shanghai, China). The cells were cultured in complete Dulbecco's modified Eagle's medium (DMEM) high glucose medium supplemented with $10 \%$ fetal bovine serum (FBS). The cells were cultured at $37^{\circ} \mathrm{C}$ under a humid atmosphere with $5 \%$ $\mathrm{CO}_{2}$. When cell confluency reached $90 \%$, cells were passaged. Cell medium was changed every two days.

RNA extraction. The lung cancer and adjacent tissues were pulverized using the liquid nitrogen grinding method. TRIzol was added with $1 \mathrm{ml} / 100 \mathrm{mg}$ tissue powders, and total RNA was extracted using the phenol chloroform method. For cell cultures, $1 \mathrm{ml}$ TRIzol was added to each $25-\mathrm{cm}^{2}$ flask when cell confluency reached $90 \%$ and total RNA was extracted using the above method. RNA was reverse transcribed into cDNA according to the instructions provided by the TIANScript RT kit.

Quantitative PCR. The expression levels of miR-138 in NSCLC and adjacent tissues and lung cancer cell lines were determined by qRT-PCR using U6 as the internal standard. The reaction system was as follow: $5 \mu \mathrm{l}$ cDNA, $10 \mu \mathrm{l}$ Mix and

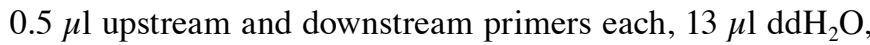
and the total volume was $30 \mu \mathrm{l}$. miR-138 upstream primer was 5'-AGCTGGTGTTGTGAATCAG-3', and the downstream primer was a universal primer provided with the miRNA cDNA kit (Takara Biotechnology, Co., Ltd., Dalian, China). The U6 primer was as follows: forward, CTCGCTTCGG CAGCACA and reverse, AACGCTTCACGAAYYYGCGT. The reaction procedure was as follows: pre-denaturation at $95^{\circ} \mathrm{C}$ for $10 \mathrm{~min}$ and 40 cycles of denaturation at $95^{\circ} \mathrm{C}$ for $30 \mathrm{sec}$ and annealing at $60^{\circ} \mathrm{C}$ for $30 \mathrm{sec}$. Each sample was measured in triplicate. The expression of miR-138 was calculated using $\Delta \Delta \mathrm{CT}$ method.

Transfection of miR-138 mimics. A549 and Calu-3 cells were cultured in DMEM medium containing 10\% FBS without antibiotics. According to different treatments, cells were divided into miR-negative control (miR-NC) and miR-138 mimics groups. When cell confluency reached $70-90 \%$, they were transfected with miR-NC and miR-138 mimics; $25 \mathrm{pm}$ miR-NC and miR-138 mimics were respectively mixed with $1 \mu \mathrm{l}$ Lipofectamine 2000, and then were added into EP tubes containing $50 \mu \mathrm{l}$ Opti-MEM medium. After standing for $5 \mathrm{~min}$, each tube was mixed and stood for $15 \mathrm{~min}$ at room temperature, and then added to the wells of the culture plate. The medium was replaced after $6 \mathrm{~h}$ and continued to culture in fresh DMEM medium with 10\% FBS.

Infection of shRNA-Sirtl by packaged lentiviral particles. Packaged lentiviral particles containing shRNA-Sirt1 were constructed and used for Sirt1 inhibition. Briefly, A549 and Calu-3 cells, divided into NC and shRNA-Sirt1 groups, were 
cultured in DMEM medium containing 10\% FBS without antibiotics. When cell confluency reached 70-90\%, they were passaged and seeded in a 24-well plate at a concentration of $5 \times 10^{4} / \mathrm{ml}$. Diluted lentivirus was added at a MOI value of 20. After $12 \mathrm{~h}$, the medium was replaced with fresh DMEM medium containing $10 \%$ FBS. After $72 \mathrm{~h}$, puromycin was added at a concentration of $1 \mu \mathrm{g} / \mathrm{ml}$ and cultured for 3 days. After that, normal medium was used. The cells were observed under a microscope for green fluorescence to analyze the infection efficiency and kept for follow-up tests.

CCK- 8 assay. After transfection of miR-NC and miR-138 for $48 \mathrm{~h}$, the cells of each group were seeded in a 96-well plate at a concentration of 5,000 cells/well. CCK-8 was added to determine the proliferation of cells in each group at $0,24,48$ and $72 \mathrm{~h}$. Three wells were used for each group and the experiment was performed in triplicate.

Flow cytometry. After digestion, each group of cells was washed twice with pre-cooled phosphate-buffered saline (PBS). The experiment was performed strictly according to the manufacturer's instruction of cell cycle assay kit (BD Biosciences, Franklin Lakes, NJ, USA). The cell cycle was detected by a flow cytometer (BD Biosciences) and the percentages of G1, S and G2/M phase cells were calculated.

Transwell assay. After digestion, each group of transfected cells was washed with PBS once, centrifuged at $500 \mathrm{x} \mathrm{g}$ for $5 \mathrm{~min}$ and then re-suspended in serum-free RPMI-1640 medium. After cell counting, $200 \mu 1$ cell suspensions were seeded in the upper wells of Matrigel-free Transwells (Corning Costar Corp., Cambridge, MA, USA) and Matrigel-coated Transwells (ECM220; Merck Millipore, Darmstadt, Germany) at a concentration of $1 \times 10^{5} /$ well. To the lower wells, $600 \mu \mathrm{l}$ RPMI-1640 medium containing 10\% FBS was added. After $24 \mathrm{~h}$ of incubation, the cells in the upper wells were removed. The number of cells that translocated the wells was analyzed with Giemsa staining. The translocated cells were counted in 5 views under a microscope and the average was used for statistical analysis.

Western blot analysis. Each group of cells was washed with pre-cooled PBS twice and added with RIPA lysis buffer and PMSF. Proteins were extracted and separated with $10 \%$ gel by SDS-PAGE. The proteins were then transferred onto a PVDF membrane. The membrane was blocked with $50 \mathrm{~g} / 1$ skim milk at room temperature for $1 \mathrm{~h}$. After blocking, primary antibodies at appropriate concentrations (Sirt 1:1,000; vimentin 1:1,000; E-cadherin 1:1,000; AMPK 1:1,000; p-AMPK 1:1,000; mTOR 1:1,000; p-mTOR 1:1,000; LC3 1:1,000; p62 1:2,000; and GAPDH 1:5,000) were added and incubated overnight on a shaker at $4^{\circ} \mathrm{C}$. After rinsing with PBS, HRP-labeled secondary antibodies were added (goat anti-mouse 1:3,000 and goat antirabbit 1:3,000) and incubated at room temperature for $1 \mathrm{~h}$. The membrane was developed using ECL reagent.

Dual-Luciferase reporter assay. According to bioinformatics predictions, the wild-type and mutant type of miR-138 binding sequences on the 3'-UTR region of Sirt1 gene were synthesized in vitro. The cleavage sites of Spe-1 and HindIII were added respectively to both ends and cloned into the pMIR-Report luciferase reporter plasmid. Then, plasmids were transfected into HEK293T cells together with miR-138 mimics using the liposome method. After $24 \mathrm{~h}$ of incubation, the cells were lysed according to the instruction of Promega Dual-Luciferase reporter system kit, and the fluorescence was determined by GloMax 20/20 luminometer (Promega, Madison, WI, USA). Renilla fluorescence was used as the internal standard, and the fluorescence of each group was statistically analyzed.

Observation of autophagosome in lung cancer cells by confocal microscope. The cells were treated with $4 \mathrm{mmol} / \mathrm{l}$ metformin (an AMPK activator) for $4 \mathrm{~h}$ to induce autophagy. Autophagy LC3B double-labeling adenovirus was constructed and packaged by Han Heng Biotech Co., with a titer of $1 \times 10^{12}$. The diluted virus was inoculated at MOI of 20 to each group of lung cancer cells. After $12 \mathrm{~h}$ of transfection, the medium was replaced and after $72 \mathrm{~h}$ of incubation, the formation of autophagosome and autophagy lysosome in each group was observed under a confocal microscope.

Statistical analysis. The statistical analysis was performed with the statistical software SPSS 16.0. The data are expressed as mean \pm standard deviation (SD). The t-test was used for comparisons between groups, while analysis of variance was used for comparison between more than three groups. A $\mathrm{P}<0.05$ was considered as statistically significant.

\section{Results}

Expression of miR-138 in NSCLC tissues and cell lines. To determine the expression of miR-138 in lung cancer tissues, quantitative PCR was performed. The results showed that miR-138 expression in NSCLC tissues obviously decreased compared to adjacent tissues, and the difference had statistical significance $(\mathrm{P}<0.001$; Fig. $1 \mathrm{~A})$. In addition, the miR-138 expression in $\mathrm{N} 1$ was significantly lower than that in N0 $(\mathrm{P}<0.01$; Fig. 1B). Additionally, the miR-138 expression level in the low differentiation group was lower $(\mathrm{P}<0.05$; Fig. 1C). Notably, there was no obvious change for the expression of miR-138 as the clinical stage TNM increased. This result indicated that the expression of miR-138 in the lung cancer tissues was obviously lower than that in the adjacent tissues and correlated to the metastasis and differentiation degree. The expressions of miR-138 in A549, Calu-3, PAa, SK-MES-1, NCl-H292, NCl-H1299, NCl-H1650 and NCl-H1975 lung cancer cell lines were further evaluated, and it was found that the expression of miR-138 in lung cancer cell lines were generally lower than normal lung epithelial tissues, while that in SK-MES-1 slightly increased (P<0.01; Fig. 1D). These results indicate that miR-138 might be involved in the differentiation, invasion and metastasis of lung cancer cells.

miR-138 inhibits A549 and Calu-3 proliferation, invasion and metastasis in vitro. In order to further investigate the biological functions of miR-138 in lung cancer, CCK-8, cell cycle analysis and Transwell assay were performed to evaluate the effects of miR-138 on the proliferation, invasion and metastasis of lung cancer cell lines. A549 and Calu-3, which showed the lowest miR-138 expression level, were selected for the following 

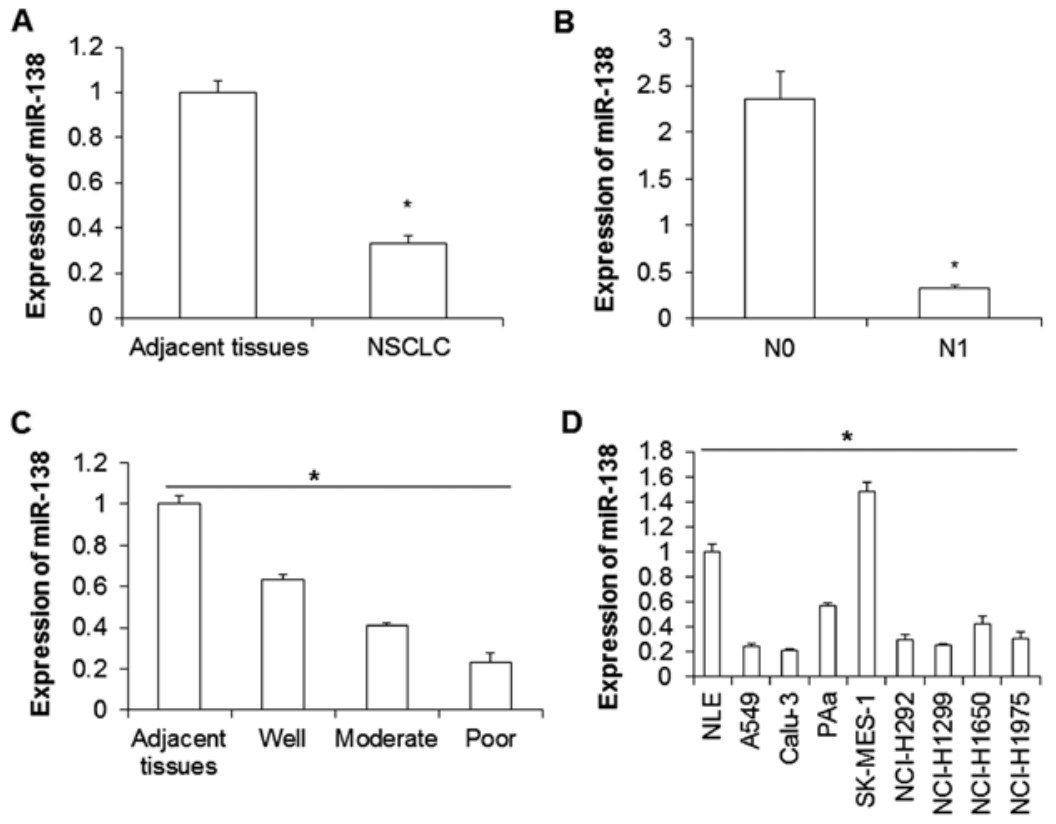

Figure 1. The expression of miR-138 in NSCLC cancer tissues determined by qRT-PCR. (A) The miR-138 expression in NSCLC tissues was obviously lower than that in the adjacent tissues ( $\mathrm{P}<0.001)$. (B) The miR-138 expression in $\mathrm{N} 1$ was significantly lower than that in N0 ("P<0.01). (C) The miR-138 expression in the NSCLC tissues with various differentiation levels was lower than the adjacent tissues ( $\mathrm{P}<0.05)$. (D) The expression of miR-138 in the lung cancer cell lines were generally lower than that in normal lung epithelial tissues, except SK-MES-1 cells ( $\mathrm{P}<0.01)$.

experiments. miR-NC and miR-138 mimics were transfected into cells. As expected, the expression of miR-138 increased significantly after transfection (Fig. 2A). Overexpression of miR-138 significantly inhibited the proliferation of A549 and Calu-3 cells $(\mathrm{P}<0.01$; Fig. 2B). Cell cycle analysis showed that overexpression of miR-138 obviously arrested A549 and Calu-3 cells at G1/S phase ( $\mathrm{P}<0.01$; Fig. $2 \mathrm{C})$. Additionally, Transwell assay indicated that overexpression of miR-138 simultaneously inhibited the metastasis and invasion abilities of A549 and Calu-3 cells ( $\mathrm{P}<0.01$; Fig. 2D). In summary, miR-138 inhibited the proliferation, invasion and migration of lung cancer cells.

Sirtl is the target gene of miR-138. The functions of miRNA molecules in the occurrence and development of a tumor are closely related to the target genes (22). To further understand the molecular mechanism underlying the role of miR-138 in NSCLC, online bioinformatics software TargetScan (www. targetscan.org) was used to predict its target gene. Results showed that there was miR-138 binding site in the 3'-UTR region of Sirt1. The wild-type and mutant type of miR-138 binding sequence is shown in Fig. 3A. Then, western blot analysis was carried out to detect levels of proteins involved in AMPK-mTOR signaling pathway and tumor cell EMT. Results showed that Sirt1 expression in A549 and Calu-3 cells obviously decreased after transfected with miR-138 mimics (Fig. 3B and C). Moreover, the phosphorylation level of the 2488 site of mTOR protein decreased obviously. The EMT of lung cancer cells was obviously inhibited, which was represented by the upregulation of E-cadherin and downregulation of vimentin (Fig. 3B and C), suggesting that AMPK-mTOR signaling pathway and tumor cell EMT may be inhibited. It has been reported that Sirt1 can activate the AMPK signaling pathway (23). To further confirm whether miR-138 directly regulate Sirtl expression, the Dual-Luciferase reporter assay was conducted. The result showed that the wild-type plasmid could bind to miR-138, while the mutant plasmid could not, indicating that miR-138 can directly regulate Sirt1 expression (Fig. 3D). In addition, the expression of 15 pairs of miR-138 and Sirt1 mRNA in NSCLC tissues was analyzed by qRT-PCR, and it was found that there was negative correlation between miR-138 and Sirt1 ( $\mathrm{P}<0.05$; Fig. 3E). In summary, it is hypothesized that miR-138 may be involved in the regulation of Sirtl-AMPK-mTOR signaling pathway and functions as a tumor suppressor.

Downregulation of Sirt1 inhibits the proliferation, invasion and migration of cancer cells. A549 and Calu-3 cells were transfected with lentivirus containing Sirt1 interference sequence to inhibit Sirt1 expression. Then, cell proliferation, invasion and migration were measured. The results showed that lentivirus transfection efficiency reached $>90 \%$ (Fig. 4A). Western blot result indicated that Sirt1 expression level obviously declined. The expression of EMT marker vimentin decreased, while that of E-cadherin enhanced (Fig. 4B). CCK-8 analysis showed that downregulation of Sirt1 inhibited lung cancer cell proliferation $(\mathrm{P}<0.05$; Fig. $4 \mathrm{C})$. Cell cycle analysis result showed that interference of Sirt1 led to G1/S phase arrest $(\mathrm{P}<0.05$; Fig. $4 \mathrm{D})$. Transwell result showed that downregulation of Sirtl expression inhibited the invasion and migration abilities of lung cancer cells $(\mathrm{P}<0.05$; Fig. 4E). At the molecular level, obvious activity decrease of the AMPKmTOR signaling pathway was observed. As downregulation of Sirtl protein, the phosphorylation level of AMPK $\alpha$ subunit decreased and the level of p-mTOR 2448 increased (Fig. 4F). Thus, these results indicate that Sirtl plays its biological functions in lung cancer cells possibly through regulating AMPK-mTOR signaling pathway. 

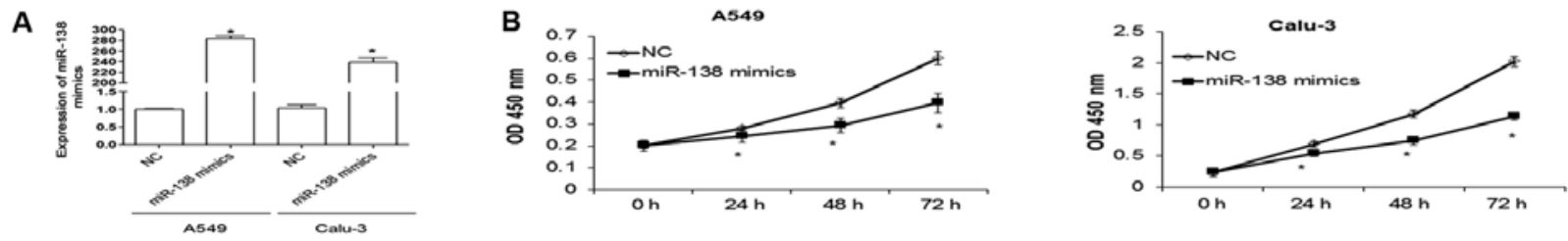

C
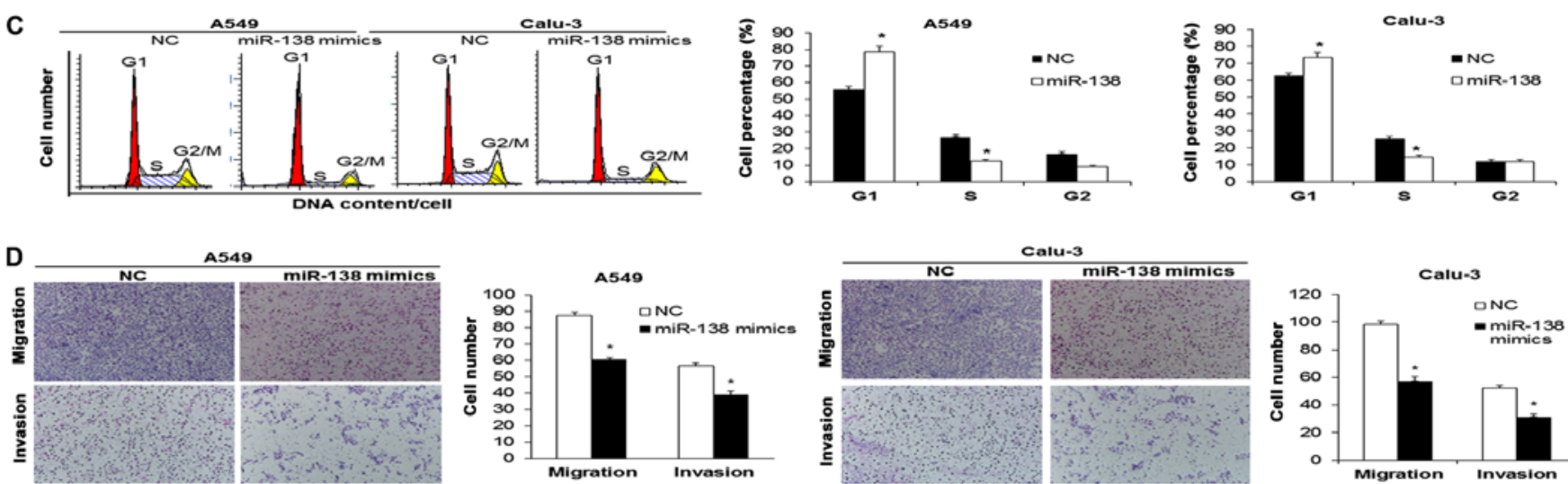

Figure 2. The effects of miR-38 on the proliferation, cell cycle, invasion and metastasis of lung cancer cells. (A) The expression changes of miR-138 in A549 and Calu- 3 cells after transfection. Compared with NC, ${ }^{*} \mathrm{P}<0.05$. (B) The effect of miR-138 on the proliferation of A549 cells and Calu-3 cells determined by CCK-8 assay. Compared with NC at 24, 48 and $72 \mathrm{~h},{ }^{*} \mathrm{P}<0.05$. (C) The effect of miR-138 on the cell cycle of A549 cells and Calu-3 cells determined by flow cytometry. Compared with NC, ${ }^{*} \mathrm{P}<0.05$. (D) The effect of miR-138 on the invasion and metastasis of A549 cells and Calu-3 cells determined by Transwell assay. The number of migrated and invaded cells was counted. Compared with $\mathrm{NC},{ }^{*} \mathrm{P}<0.05$.

A

$$
\begin{array}{ccc}
\text { Wild-type } & 5^{\prime} \text {...GUACAGGAAUUGUUC--CACCAGCA.... } \\
& 3^{\prime} \quad \text { GCCGGACUAAGUGUUGUGGUCGA } \\
& & \\
\text { Mutant type } & 5^{\prime} \text {...GUACAGGAAUUGUUC--CACCAGCA... } \\
& 3^{\prime} & \text { GCCGGACUGAGUGUUGGGAUAA }
\end{array}
$$

B

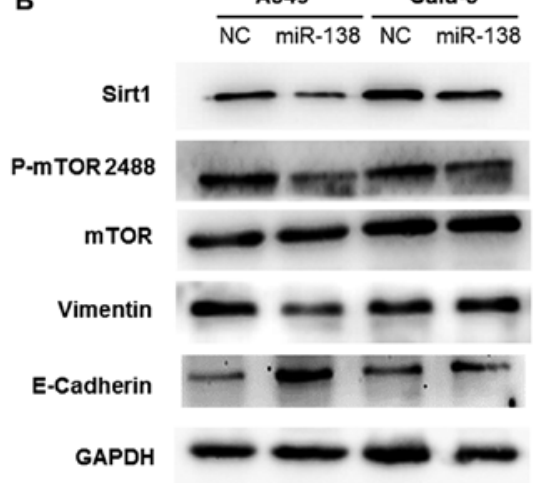

D

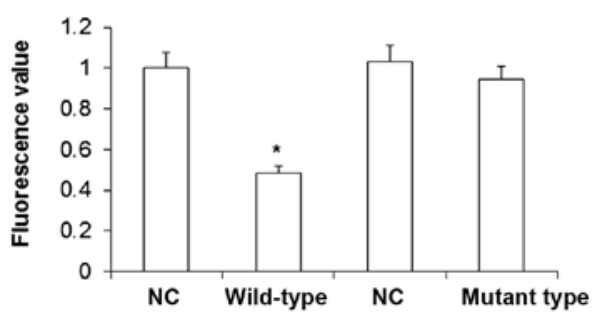

$\mathbf{E}$
C
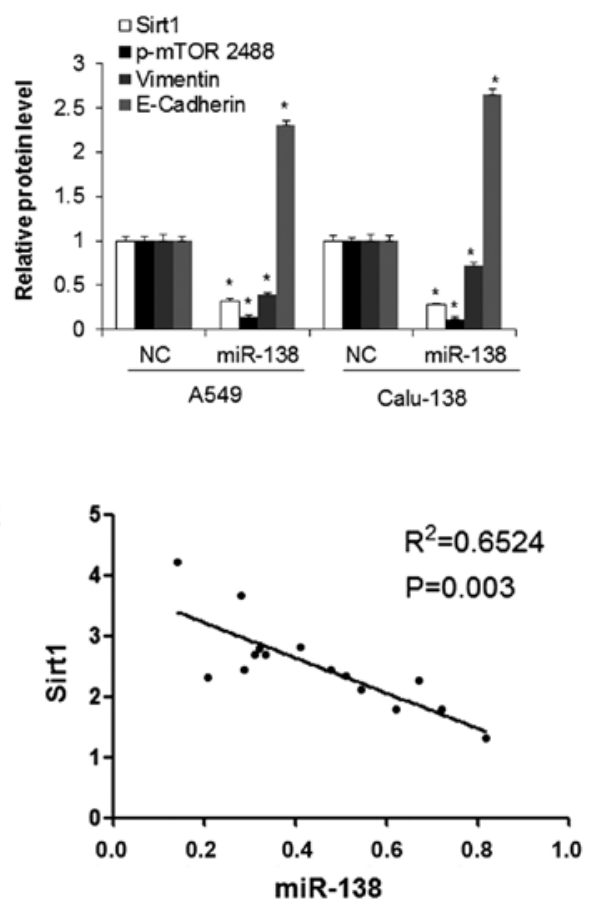

Figure 3. The effects of miR-138 transfection. (A) The wild-type and mutant type of miR-138 binding sequence. (B) The expression of Sirt1, phosphorylated and non-phosphorylated mTOR and the EMT markers, E-cadherin and vimentin after transfected with miR-138 mimics. (C) Quantification of the protein bands in (B). Compared with corresponding NC, ${ }^{*} \mathrm{P}<0.05$. (D) Relative fluorescence value by Dual-Luciferase reporter assay. Compared with NC, ${ }^{*} \mathrm{P}<0.05$. (E) Correlation between miR-138 and Sirt1 mRNA in NSCLC tissues. 

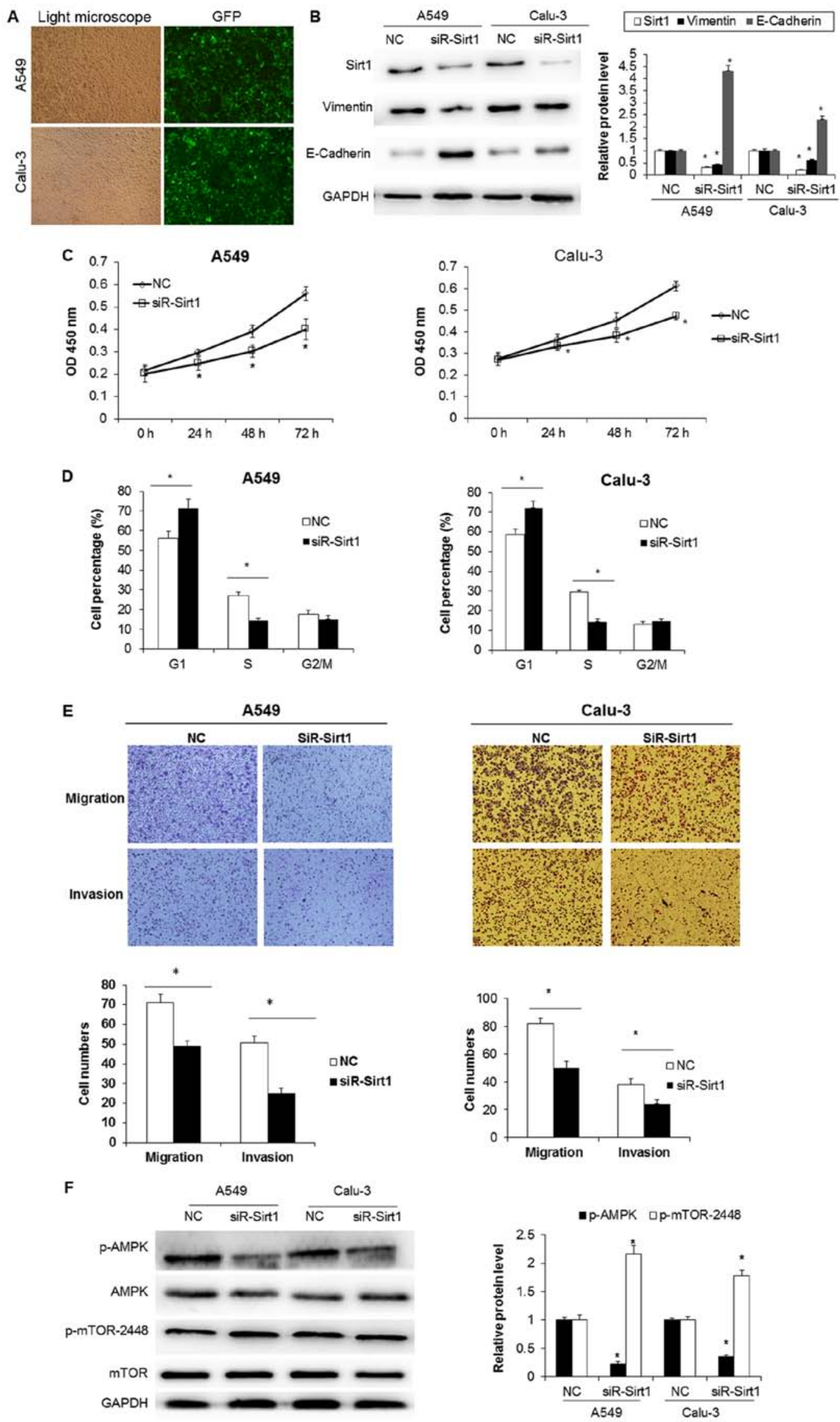

Figure 4. The effects of downregulation of Sirt1. (A) Detection of the GFP fluorescence to determine the transfection efficiency. (B) The effect of Sirt1 knockdown on the EMT markers, vimentin and E-cadherin, analyzed by western blot analysis. Compared with corresponding NC, ${ }^{*} \mathrm{P}<0.05$. (C) The effect of Sirt1 knockdown on the proliferation of A549 and Calu-3 cells determined by CCK-8 assay. Compared with NC at 24,48 and $72 \mathrm{~h},{ }^{*} \mathrm{P}<0.05$. (D) The effect of Sirt1 knockdown on the cell cycles of A549 and Calu-3 cells analyzed by flow cytometry. "P<0.05. (E) The effect of Sirt1 knockdown on the invasion and metastasis of A549 and Calu-3 cells. Compared with NC, ${ }^{*} \mathrm{P}<0.05$. (F) The expression of AMPK-mTOR-related proteins in A549 and Calu-3 cells determined by western blot analysis. Compared with corresponding NC, ${ }^{*} \mathrm{P}<0.05$. 

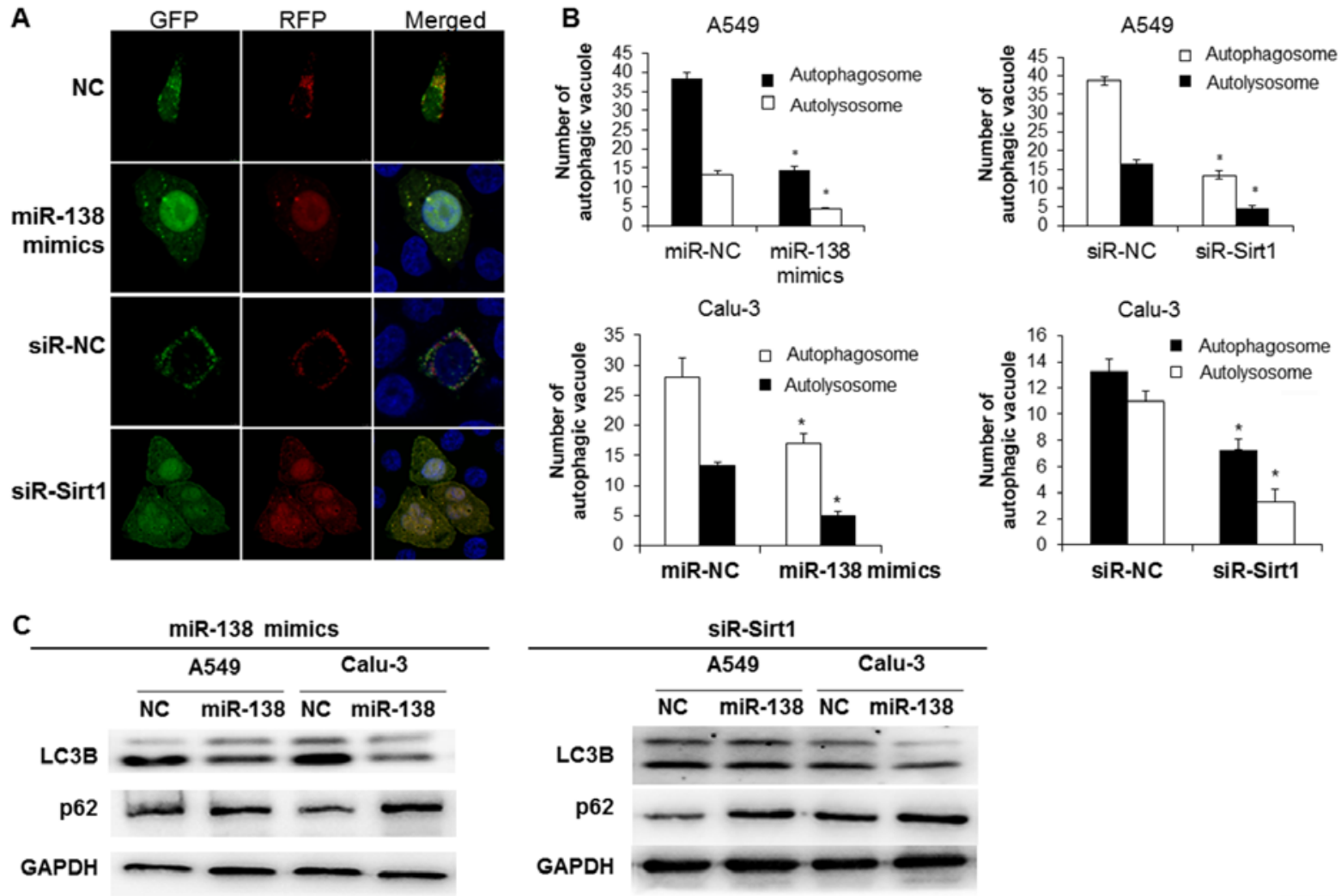

Figure 5. Inhibition of the autophagy of A549 and Calu-3 cells by overexpression of miR-138 and downregulation of Sirt1. The cells were treated with $4 \mathrm{mmol} / 1$ metformin for $4 \mathrm{~h}$ to induce autophagy. (A) Observation of the autophagosome and autolysosome in lung cancer cells by confocal microscope. (B) The number of autophagosome and autolysosome in A549 and Calu-3 cells after overexpression of miR-138 and downregulation of Sirt1. Compared with NC, ${ }^{*} \mathrm{P}<0.05$. (C) Effects of miR-138 and Sirt1 on the expression of autophagy related proteins detected by western blot analysis. Compared with corresponding NC, "P<0.05.

miR-138 inhibits cell autophagy by regulating Sirt1. It has been reported that cell autophagy could be regulated by AMPK-mTOR signaling pathway (24). Results of the present study showed that miR-138 could regulate Sirt1 to inhibit AMPK signaling pathway and promote mTOR phosphorylation. The phosphorylation level of mTOR is closely related to autophagy activity (25). Therefore, we propose that miR-138 may inhibit lung cancer cell autophagy. After transfection of LC3B double labeling adenovirus for $72 \mathrm{~h}$, the autophagy of lung cancer cells was observed using a confocal microscope. The results showed that the autophagosome (green) and autophagy lysosome (red) in the miR-138 overexpressed tumor cells were both lower than the negative control group (Fig. 5A and B). In addition, downregulation of Sirt1 gene expression could also obviously decrease the number of autophagosome and autophagy lysosomes in lung cancer cells (Fig. 5A and B). Moreover, western blot results showed that miR-138 overexpression and downregulation of Sirt1 respectively inhibited the II/I conversion ratio of LC3B and upregulated $\mathrm{p} 62$ protein expression (Fig. 5C). Therefore, these results demonstrate that miR-138 may inhibit lung cancer cell autophagy by regulating Sirt1 expression.

\section{Discussion}

In the present study, we found that miR-138 expression in NSCLC was obviously decreased and negatively correlated to tumor differentiation and lymph node metastasis. In vitro experiments verified that miR-138 functioned as a tumor suppressor gene, and Sirt1 was one of its target genes. miR-138 could downregulate Sirt1 expression, inhibit EMT, inactivate AMPK-mTOR, and thus inhibit autophagy of lung cancer cells, leading to obvious inhibition of cell proliferation, invasion and migration of lung cancer cells.

Studies have shown that miR-138 was downregulated in many types of cancer and that it could inhibit the occurrence and development of tumors. For example, Xiao et al (26) indicated that miR-138 could target and regulate the expression of YAP1 gene, and thus inhibit the invasion and metastasis of lung cancer cells. In addition, its reduced expression was one of the reasons promoting metastasis and recurrence of osteosarcoma. Sun et al (27) found that miR-138 inhibited tumor cell metastasis by decreasing bladder cancer EMT. In NSCLC, it was reported that miR-138 could regulate the resistance of tumor cells to chemotherapy by suppressing EMT (28). Recently, Tan et al (29) reported that miR-138 inhibited migration and invasion of NSCLC through targeting LIMK1. The above research indicated that miR-138 plays a role of inhibitor in the development of tumors. In accordance with these studies, we also found that miR-138 expression was down regulated in NSCLC tissues and cell lines, and negatively correlated to the differentiation degree and lymph node metastasis. In vitro, we also found that miR-138 inhibited the proliferation and metastasis of lung cancer cells by CCK-8 and Transwell experiments, demonstrating that miR-138 functions as a tumor suppressor gene in NSCLC. However, the expression 
level of miR-138 was slightly increased in SK-MES-1 cell line. This may be due to different cancer cell lines having different genetic backgrounds. Studies are needed to further clarify this discrepancy. To avoid this, in the following experiments, the cell line with the lowest miR-138 expression was used.

miRNA exerts biological function by regulating target genes. We found that Sirt1 might be a target gene of miR-138 by bioinformatics tools. By Dual-Luciferase reporter assay and western blot analysis, we confirmed that the expression of miR-138 was negatively related with Sirt1 mRNA in NSCLC tissues, demonstrating that miR-138 could directly bind to the 3'-UTR of Sirt1 mRNA. Sirt1 is a NAD ${ }^{+}$-dependent histone deacetylase widely distributed intracellularly and extracellularly. It plays important roles in cell proliferation, apoptosis, aging, inflammation and metabolism (30-32). In addition, it can affect tumorigenesis, development and metastasis of a tumor $(33,34)$. Sirt1 is heterogeneously expressed in tumor, which is closely related to tumor types. In some tumors, Sirt1 is highly expressed, while in others, its expression is obviously inhibited $(35,36)$. Existing studies have shown that Sirt1 functions promoting tumor growth. For example, Sirt1 can interact with p53 and cause deacetylation of Lys 382 at the C-terminal of p53. Thus, p53 is inactivated and a series of tumor suppressor functions mediated by $\mathrm{p} 53$, such as transcription activation, is inhibited (37). In epigenetics, Sirtl can influence many tumor suppressor genes and can also affect the activities of DNA repair-related proteins (38-43). In addition, inhibition of Sirt1 expression and activity can inhibit the growth, invasion and metastasis of many tumors (44). In the present study, it was found that downregulation of Sirt1 inhibited the proliferation and metastasis of A549 and Calu-3.

Recent research has shown that Sirtl can activate AMPK signaling pathway, which is closely related to the activation of mTOR $(45,46)$. We also found that upregulation of miR-138 or downregulation of Sirt1 inhibited AMPK-mTOR pathway, indicating that miR-138 may regulate AMPK-mTOR pathway. Convincing evidence showed that AMPK-mTOR pathway is important in regulating autophagy $(25,47)$. Activation of AMPK is capable of inhibiting the phosphorylation of mTOR, and thus promotes cell autophagy $(24,48)$. Autophagy is closely related to tumor metastasis, EMT, apoptosis and drug resistance (49-51). For instance, autophagy was able to degrade the epithelial marker E-cadherin and promoted EMT of tumors $(52,53)$. Inhibition of autophagy could promote apoptosis of tumor cells (54). To date, whether Sirt1 regulates autophagy of NSCLC is unclear. The present study found that overexpressed miR-138, or decreased Sirt1, both inhibited autophagy of NSCLC cells. Overexpression of Sirt1 could rescue the autophagy inhibition induced by miR-138, suggesting that miR-138 regulate the autophagy of NSCLC by interaction with Sirt1.

In conclusion, miR-138 can target and regulate Sirt 1 to downregulate the activity of AMPK signaling pathway, promote the phosphorylation levels of mTOR, decrease the autophagy activity of lung cancer cells, and thus inhibit the occurrence and development of tumor cells. Declined expression of miR-138 in NSCLC tissues could be one of the molecular mechanisms leading to tumor recurrence and metastasis. miR-138 may be used as a potential new target for lung cancer treatment.

\section{Acknowledgements}

The present study was supported by the Program of Science Technology Department of Zhejiang Province (no. 2016C33SAB0009), and the Program of Science and Technology Bureau of Lishui City (no. 2014JYZB12).

\section{References}

1. Constanzo JD, Tang KJ, Rindhe S, Melegari M, Liu H, Tang X, Rodriguez-Canales J, Wistuba I and Scaglioni PP: PIAS1-FAK interaction promotes the survival and progression of non-small cell lung cancer. Neoplasia 18: 282-293, 2016.

2. Moores SL, Chiu ML, Bushey BS, Chevalier K, Luistro L, Dorn K, Brezski RJ, Haytko P, Kelly T, Wu SJ, et al: A novel bispecific antibody targeting EGFR and cMet is effective against EGFR inhibitor-resistant lung tumors. Cancer Res 76: 3942-3953, 2016.

3. Casaluce F, Sgambato A, Sacco PC, Palazzolo G, Maione P, Rossi A, Ciardiello F and Gridelli C: Resistance to crizotinib in advanced non-small cell lung cancer (NSCLC) with ALK rearrangement: mechanisms, treatment strategies and new targeted therapies. Curr Clin Pharmacol 11: 77-87, 2016.

4. Shen H, Cao Y, Li X, Tan Y, Chen J, Yang Z, Kong Y and Yuan Y: Surgical intervention improves survival for metastatic non-small cell lung cancer patients. Medicine (Baltimore) 95: e3800, 2016

5. Zhou J, Song XB, He H, Zhou Y, Lu XJ and Ying BW: Prevalence and clinical profile of EGFR mutation in non-small-cell lung carcinoma patients in Southwest China. Asian Pac J Cancer Prev 17: 965-971, 2016.

6. Zequn N, Xuemei Z, Wei L, Zongjuan M, Yujie Z, Yanli H, Yuping Z, Xia M, Wei W, Wenjing D, et al: The role and potential mechanisms of LncRNA-TATDN1 on metastasis and invasion of non-small cell lung cancer. Oncotarget 7: 18219-18228, 2016.

7. Wang B, Ding W, Zhang M, Li H, Guo H, Lin L, Chen J and $\mathrm{Gu}$ Y: Role of FOXO1 in aldosterone-induced autophagy: A compensatory protective mechanism related to podocyte injury. Oncotarget 7: 45331-45351, 2016.

8. Bah A, Lacarrière $\mathrm{C}$ and Vergne I: Autophagy-related proteins target ubiquitin-free mycobacterial compartment to promote killing in macrophages. Front Cell Infect Microbiol 6: 53, 2016.

9. Barnard RA, Regan DP, Hansen RJ, Maycotte P, Thorburn A and Gustafson DL: Autophagy inhibition delays early but not late stage metastatic disease. J Pharmacol Exp Ther 358: 282-293, 2016.

10. Song L, Liu S, Zhang L, Yao H, Gao F, Xu D and Li Q: MiR-21 modulates radiosensitivity of cervical cancer through inhibiting autophagy via the PTEN/Akt/HIF-1 $\alpha$ feedback loop and the Akt-mTOR signaling pathway. Tumour Biol 37: 12161-12168, 2016.

11. Li Q, Liang X, Wang Y, Meng X, Xu Y, Cai S, Wang Z, Liu J and Cai G: miR-139-5p inhibits the epithelial-mesenchymal transition and enhances thechemotherapeutic sensitivity of colorectal cancer cells by downregulating BCL2. Sci Rep 6: 27157, 2016.

12. Momtazi AA, Derosa G, Maffioli P,Banach M and Sahebkar A: Role of microRNAs in the therapeutic effects of curcumin in non-cancer diseases. Mol Diagn Ther 20: 335-345, 2016.

13. Tomasetti M, Amati M, Santarelli L and Neuzil J: MicroRNA in metabolic re-programming and their role in tumorigenesis. Int $\mathbf{J}$ Mol Sci 17: pii: E754, 2016.

14. Azizian A, Gruber J, Ghadimi BM and Gaedcke J: MicroRNA in rectal cancer. World J Gastrointest Oncol 8: 416-426, 2016.

15. Bao L, Lv L, Feng J, Chen Y, Wang X, Han S and Zhao H: miR-487b-5p regulates temozolomide resistance of lung cancer cells through LAMP2-medicated autophagy. DNA Cell Biol 35: 385-392, 2016.

16. Tomasetti M, Staffolani S, Nocchi L, Neuzil J, Strafella E, Manzella N, Mariotti L, Bracci M, Valentino M, Amati M, et al: Clinical significance of circulating miR-126 quantification in malignant mesothelioma patients. Clin Biochem 45: 575-581, 2012.

17. Nyhan MJ, O'Donovan TR, Boersma AW, Wiemer EA and McKenna SL: MiR-193b promotes autophagy and non-apoptotic cell death in oesophageal cancer cells. BMC Cancer 16: 101, 2016. 
18. Li J, Xia W, Su X, Qin X, Chen Y, Li S, Dong J, Ding H, Li H, Huang A, et al: Species-specific mutual regulation of p53 and miR-138 between human, rat and mouse. Sci Rep 6: 26187, 2016.

19. Jiang B, Mu W, Wang J, Lu J, Jiang S, Li L, Xu H and Tian H: MicroRNA-138 functions as a tumor suppressor in osteosarcoma by targeting differentiated embryonic chondrocyte gene 2. J Exp Clin Cancer Res 35: 69, 2016.

20. Li B, Yang XX, Wang D and Ji HK: MicroRNA-138 inhibits proliferation of cervical cancer cells by targeting c-Met. Eur Rev Med Pharmacol Sci 20: 1109-1114, 2016.

21. Melloni G, Muriana P, Bandiera A, Fontana R, Maggioni D, Russo V, Doglioni C and Zannini P: Prognostic role of liver X receptor-alpha in resected stage II and III non-small-cell lung cancer. Clin Respir J: Jul 12, 2016 (Epub ahead of print). doi: 10.1111/crj.12522.

22. Qin Q, Wei F, Zhang J, Wang X and Li B: miR-134 inhibits non-small cell lung cancer growth by targeting the epidermal growth factor receptor. J Cell Mol Med 20: 1974-1983, 2016.

23. Talero E, Alcaide A, Ávila-Román J, García-Mauriño S, Vendramini-Costa D and Motilva V: Expression patterns of sirtuin 1-AMPK-autophagy pathway in chronic colitis and inflammation-associated colon neoplasia in IL-10-deficient mice. Int Immunopharmacol 35: 248-256, 2016

24. He Q, Sha S, Sun L, Zhang J and Dong M: GLP-1 analogue improves hepatic lipid accumulation by inducing autophagy via AMPK/mTOR pathway. Biochem Biophys Res Commun 476 : 196-203, 2016.

25. Ji L, Li L, Qu F, Zhang G, Wang Y, Bai X, Pan S, Xue D, Wang G and Sun B: Hydrogen sulphide exacerbates acute pancreatitis by over-activating autophagy via AMPK/mTOR pathway. J Cell Mol Med 20: 2349-2361, 2016.

26. Xiao L, Zhou H, Li XP, Chen J, Fang C, Mao CX, Cui JJ, Zhang W, Zhou HH, Yin JY, et al: MicroRNA-138 acts as a tumor suppressor in non small cell lung cancer via targeting YAP1. Oncotarget 7: 40038-40046, 2016.

27. Sun DK, Wang JM, Zhang P and Wang YQ: MicroRNA-138 regulates metastatic potential of bladder cancer through ZEB2. Cell Physiol Biochem 37: 2366-2374, 2015.

28. Jin Z, Guan L, Song Y, Xiang GM, Chen SX and Gao B: MicroRNA-138 regulates chemoresistance in human non-small cell lung cancer via epithelial mesenchymal transition. Eur Rev Med Pharmacol Sci 20: 1080-1086, 2016.

29. Tan Y, Hu H, Tan W, Jin L, Liu J and Zhou H: MicroRNA-138 inhibits migration and invasion of non-small cell lung cancer cells by targeting LIMK1. Mol Med Rep 14: 4422-4428, 2016.

30. Tian XF, Ji FJ, Zang HL and Cao H: Activation of the miR-34a/ SIRT1/p53 signaling pathway contributes to the progress of liver fibrosis via inducing apoptosis in hepatocytes but not in HSCs. PLoS One 11: e0158657, 2016.

31. Wang Y, Bi Y, Chen X, Li C, Li Y, Zhang Z, Wang J, Lu Y, Yu Q, $\mathrm{Su} \mathrm{H}$, et al: Histone deacetylase SIRT1 negatively regulates the differentiation ofinterleukin-9-producing $\mathrm{CD}^{+}{ }^{+} \mathrm{T}$ cells. Immunity 44: 1337-1349, 2016.

32. Ye Z, Fang J, Dai S, Wang Y, Fu Z, Feng W, Wei Q and Huang P. MicroRNA-34a induces a senescence-like change via the downregulation of SIRT1 and up-regulation of p53 protein in human esophageal squamous cancer cells with a wild-type p53 gene background. Cancer Lett 370: 216-221, 2016.

33. Liu L, Liu C, Zhang Q, Shen J, Zhang H, Shan J, Duan G, Guo D, Chen X, Cheng J, et al: SIRT1-mediated transcriptional regulation of SOX2 is important for self-renewal of Liver cancer stem cells. Hepatology 64: 814-827, 2016.

34. Shen ZL, Wang B, Jiang KW, Ye CX, Cheng C, Yan YC, Zhang JZ, Yang Y, Gao ZD, Ye YJ, et al: Downregulation of miR-199b is associated with distant metastasis in colorectal cancer via activation of SIRT1 and inhibition of CREB/KISS1 signaling. Oncotarget 7: 35092-35105, 2016.

35. Huffman DM, Grizzle WE, Bamman MM, Kim JS, Eltoum IA, Elgavish A and Nagy TR: SIRT1 is significantly elevated in mouse and human prostate cancer. Cancer Res 67: 6612-6618, 2007.
36. Stünkel W, Peh BK, Tan YC, Nayagam VM, Wang X, SaltoTellez M, Ni B, Entzeroth M and Wood J: Function of the SIRT1 protein deacetylase in cancer. Biotechnol J 2: 1360-1368, 2007.

37. Vaziri H, Dessain SK, Ng Eaton E, Imai SI, Frye RA, Pandita TK, Guarente L and Weinberg RA: hSIR2 $2^{\text {SIRT1 }}$ functions as an NAD-dependent p53 deacetylase. Cell 107: 149-159, 2001.

38. Leung WK, Wu MS, Kakugawa Y, Kim JJ, Yeoh KG, Goh KL, Wu KC, Wu DC, Sollano J, Kachintorn U, et al; Asia Pacific Working Group on Gastric Cancer: Screening for gastric cancer in Asia: Current evidence and practice. Lancet Oncol 9: 279-287, 2008.

39. Motta MC, Divecha N, Lemieux M, Kamel C, Chen D, Gu W, Bultsma Y, McBurney M and Guarente L: Mammalian SIRT1 represses forkhead transcription factors. Cell 116: 551-563, 2004.

40. Wang C, Chen L, Hou X, Li Z, Kabra N, Ma Y, Nemoto S Finkel T, Gu W, Cress WD, et al: Interactions between E2F1 and SirT1 regulate apoptotic response to DNA damage. Nat Cell Biol 8: 1025-1031, 2006.

41. Jeong J, Juhn K, Lee H, Kim SH, Min BH, Lee KM, Cho MH, Park GH and Lee KH: SIRT1 promotes DNA repair activity and deacetylation of Ku70. Exp Mol Med 39: 8-13, 2007.

42. Dai JM, Wang ZY, Sun DC, Lin RX and Wang SQ: SIRT1 interacts with $\mathrm{p} 73$ and suppresses p73-dependent transcriptional activity. J Cell Physiol 210: 161-166, 2007.

43. Wong S and Weber JD: Deacetylation of the retinoblastoma tumour suppressor protein by SIRT1. Biochem J 407: 451-460, 2007.

44. George $\mathrm{J}$ and Ahmad N: Mitochondrial sirtuins in cancer: Emerging roles and therapeutic potential. Cancer Res 76: 2500-2506, 2016

45. Salminen A, Kaarniranta K and Kauppinen A: AMPK and HIF signaling pathways regulate both longevity and cancer growth: the good news and the bad news about survival mechanisms. Biogerontology 17: 655-680, 2016.

46. Han X, Tai H, Wang X, Wang Z, Zhou J, Wei X, Ding Y, Gong H, Mo $\mathrm{C}$, Zhang $\mathrm{J}$, et al: AMPK activation protects cells from oxidative stress-induced senescence via autophagic flux restoration and intracellular $\mathrm{NAD}^{+}$elevation. Aging Cell 15: 416-427, 2016.

47. Gong X, Wang H, Ye Y,Shu Y, Deng Y, He X, Lu G and Zhang S: miR-124 regulates cell apoptosis and autophagy in dopaminergic neurons and protects them by regulating AMPK/mTOR pathway in Parkinson's disease. Am J Transl Res 8: 2127-2137. eCollection, 2016.

48. Mao N, Tan RZ, Wang SQ, Wei C, Shi XL, Fan JM and Wang L: Ginsenoside Rg1 inhibits angiotensin II-induced podocyte autophagy via AMPK/mTOR/PI3K pathway. Cell Biol Int 40: 917-925, 2016.

49. Pigna E, Berardi E, Aulino P, Rizzuto E, Zampieri S, Carraro U, Kern H, Merigliano S, Gruppo M, Mericskay M, et al: Aerobic exercise and pharmacological treatments counteract cachexia by modulating autophagy in colon cancer. Sci Rep: May 31, 2016. doi: 10.1038/srep26991.

50. Zhong Z, Sanchez-Lopez E and Karin M: Autophagy, inflammation, and immunity: A troika governing cancer and its treatment. Cell 166: 288-298, 2016.

51. Cao ZX, Yang YT, Yu S, Li YZ, Wang WW, Huang J, Xie XF, Xiong L, Lei S and Peng C: Pogostone induces autophagy and apoptosis involving $\mathrm{PI} 3 \mathrm{~K} / \mathrm{Akt} / \mathrm{mTOR}$ axis in human colorectal carcinoma HCT116 cells. J Ethnopharmacol 202: 20-27, 2017.

52. Pang M, Wang H, Rao P, Zhao Y, Xie J, Cao Q, Wang Y, Wang YM, Lee VW, Alexander SI, et al: Autophagy links $\beta$-catenin and Smad signaling to promote epithelial-mesenchymal transition via upregulation of integrin linked kinase. Int J Biochem Cell Biol 76: 123-134, 2016.

53. Qian HR and Yang Y: Functional role of autophagy in gastric cancer. Oncotarget 7: 17641-17651, 2016.

54. Ouyang F, Huang H, Zhang M, Chen M, Huang H, Huang F and Zhou S: HMGB1 induces apoptosis and EMT in association with increased autophagy following H/R injury in cardiomyocytes. Int J Mol Med 37: 679-689, 2016. 